\title{
INVARIANCE OF A SHIFT-INVARIANT SPACE
}

\author{
AKRAM ALDROUBI, CARLOS CABRELLI, CHRISTOPHER HEIL, \\ KERI KORNELSON, AND URSULA MOLTER
}

\begin{abstract}
A shift-invariant space is a space of functions that is invariant under integer translations. Such spaces are often used as models for spaces of signals and images in mathematical and engineering applications. This paper characterizes those shift-invariant subspaces $S$ that are also invariant under additional (noninteger) translations. For the case of finitely generated spaces, these spaces are characterized in terms of the generators of the space. As a consequence, it is shown that principal shift-invariant spaces with a compactly supported generator cannot be invariant under any non-integer translations.
\end{abstract}

\section{INTRODUCTION}

A shift-invariant space (SIS) is a space of functions that is invariant under integer translations.

They have applications throughout mathematics and engineering, as such spaces are often used as models for spaces of signals and images, see Grö01, [HW96], Mal98.

One example of a shift-invariant space is the Paley-Wiener space of functions that are bandlimited to $[-1 / 2,1 / 2]$ :

$$
P W(\mathbb{R})=\left\{f \in L^{2}(\mathbb{R}): \operatorname{supp}(\widehat{f}) \subseteq\left[-\frac{1}{2}, \frac{1}{2}\right]\right\} .
$$

This SIS has the property that it is not only invariant under integer translations, but it is in fact invariant under every real translation. A space with this property is said to be translation-invariant. A classical theorem of Fourier analysis (often attributed to Wiener, see for example [Hel64]), completely characterizes the closed translation-invariant subspaces of $L^{2}(\mathbb{R})$ as being of the form

$$
\left\{f \in L^{2}(\mathbb{R}): \operatorname{supp}(\widehat{f}) \subseteq A\right\}
$$

where $A \subseteq \mathbb{R}$ is measurable.

Date: December 21, 2008.

2000 Mathematics Subject Classification. Primary 42C40; Secondary 42C15, 46C99.

Key words and phrases. Dimension function, Fiber space, frames, Gramian operator, Range function, Riesz basis, shift-invariant space, translation-invariant space.

The research of A. Aldroubi was supported in part by NSF Grant DMS-0807464. The research of C. Cabrelli and U. Molter was partially supported by Grants FONCyT PICT 2006-00177, CONICET, PIP 5650, UBACyT X058 and X108. The research of C. Heil was supported in part by NSF Grant DMS-0806532. The research of K. Kornelson was supported in part by NSF Grant DMS0701164, Grinnell College CSFS, the Woodrow Wilson Fellowship Foundation, and the IMMERSE program at the University of Nebraska-Lincoln. 
In many applications, it is desirable to have a shift-invariant space that possesses extra invariances [CS03, Web00. In this paper we characterize those shift-invariant subspaces $S$ that are not only invariant under integer translations, but are also invariant under some particular set of translations $M \subseteq \mathbb{R}$. We show that there are only two possibilities:

- either $S$ is translation-invariant, or

- there exists an $n \in \mathbb{N}$ such that $S$ is invariant under translations by multiples of $1 / n$, but not invariant under translations by $1 / m$ with $m>n$.

We give several characterizations of those shift-invariant spaces that are $\frac{1}{n} \mathbb{Z}$-invariant. A trivial way to create such a space is to fix a function $g \in L^{2}(\mathbb{R})$ and set

$$
S=\overline{\operatorname{span}}\left\{g\left(x-\frac{k}{n}\right): k \in \mathbb{Z}\right\},
$$

the closed span of the $\frac{1}{n} \mathbb{Z}$ translates of $g$. However, we are interested in the more subtle question of recognizing when a given SIS is $\frac{1}{n} \mathbb{Z}$-invariant. For example, in many applications one is presented with a SIS of the form

$$
S=\overline{\operatorname{span}}\{g(x-k): k \in \mathbb{Z}\},
$$

and it is not obvious whether such a space possesses any invariants other than translation by integers. We completely determine the invariances of such a space in terms of properties of $g$ and, more generally, characterize any SIS that is $\frac{1}{n} \mathbb{Z}$ invariant.

One interesting corollary of our characterization is that the shift-invariant space generated by a compactly supported function is not invariant under any translations other than $\mathbb{Z}$. Thus, the shift-invariant spaces associated with compactly supported multiresolution analyses and wavelets are already "maximally invariant."

\section{Notation And Definitions}

We normalize the Fourier transform of $f \in L^{1}(\mathbb{R})$ as

$$
\widehat{f}(\omega)=\int_{-\infty}^{\infty} f(x) e^{-2 \pi i \omega x} d x .
$$

The Fourier transform extends to a unitary operator on $L^{2}(\mathbb{R})$. Given $\mathcal{F} \subseteq L^{2}(\mathbb{R})$, we set $\widehat{\mathcal{F}}=\{\widehat{f}: f \in \mathcal{F}\}$.

The translation operator $T_{a}$ is $T_{a} f(x)=f(x-a)$. Note that $\left(T_{a} f\right)^{\wedge}(\omega)=$ $e^{-2 \pi i a \omega} \widehat{f}(\omega)$.

A function $f$ is $b \mathbb{Z}$-periodic if $T_{b k} f=f$ for all $k \in \mathbb{Z}$. A set $A \subseteq \mathbb{R}$ is bZ-periodic if its characteristic function is $b \mathbb{Z}$-periodic.

A shift-invariant space (SIS) is a closed subspace $S$ of $L^{2}(\mathbb{R})$ that is invariant under integer translations. We say that $S$ is $b \mathbb{Z}$-invariant if it is invariant under translation by $b k$ for all $k \in \mathbb{Z}$.

Given $\mathcal{F} \subseteq L^{2}(\mathbb{R})$, we define

$$
\mathcal{T}_{\mathbb{Z}}(\mathcal{F})=\left\{T_{j} f: f \in \mathcal{F}, j \in \mathbb{Z}\right\} .
$$


The SIS generated by $\mathcal{F}$ is

$$
\mathfrak{S}(\mathcal{F})=\overline{\operatorname{span}}\left(\mathcal{T}_{\mathbb{Z}}(\mathcal{F})\right)=\overline{\operatorname{span}}\left\{T_{j} f: f \in \mathcal{F}, j \in \mathbb{Z}\right\} .
$$

We call $\mathcal{F}$ a set of generators for $\mathfrak{S}(\mathcal{F})$. When $\mathcal{F}=\{f\}$ consists of a single function, we simply write $\mathfrak{S}(f)$.

The length of a SIS $S$ is the minimum cardinality of the sets $\mathcal{F}$ such that $S=$ $\mathfrak{S}(\mathcal{F})$. A SIS of length one is called a principal SIS. A SIS of finite length is a finitely generated SIS.

We will write $W=U \dot{\oplus} V$ to denote the orthogonal direct sum of closed subspaces of $L^{2}(\mathbb{R})$, i.e., the subspaces $U, V$ must be closed and orthogonal, and $W$ is their direct sum.

The Lebesgue measure of a set $E \subseteq \mathbb{R}$ is denoted by $|E|$.

The cardinality of a finite set $F$ is denoted by $\# F$.

\section{ORDER OF INVARIANCE}

Let $S$ be a SIS. If $\theta$ is a real number, we will say that $S$ is invariant under translations by $\theta$ or that $S$ is $\theta$-invariant if

$$
f \in S \quad \Longrightarrow \quad T_{\theta} f \in S .
$$

We have the following Proposition.

Proposition 3.1. Let $S$ be a SIS and define,

$$
M=\{\theta \in \mathbb{R}: S \text { is } \theta \text {-invariant }\} .
$$

Then $M$ is a closed additive subgroup of $\mathbb{R}$ containing $\mathbb{Z}$.

Proof. Note that $\mathbb{Z} \subseteq M$ since $S$ is shift-invariant. To see that M is closed, let $\left\{\theta_{j}\right\}$ be a sequence in $M$ such that $\theta_{j} \rightarrow \theta$. Then, given any $f \in S$, we have,

$$
\left\|T_{\theta_{j}} f-T_{\theta} f\right\|_{2}^{2}=\int_{-\infty}^{\infty}\left|f\left(x-\theta_{j}\right)-f(x-\theta)\right|^{2} d x \rightarrow 0 \quad \text { as } j \rightarrow \infty .
$$

So, since $S$ is closed and $T_{\theta_{j}} \in S, T_{\theta} f$ must be in $S$ and therefore $\theta \in M$.

Let us prove now that $\mathrm{M}$ is indeed an additive subgroup of $\mathbb{R}$. Clearly, $\mathrm{M}$ is closed under addition. Furthermore, if $n, m \in \mathbb{Z}$ with $n>0$ and $\theta \in M$ then $n \theta+m \in M$.

We need to see that $-\theta$ is in $M$ for each $\theta \in M$. For this, let us first consider the case that $\theta$ is rational. So we can assume that $\theta=p / q$ with $q>0$.

Then, if $p / q \in M$ we have

$$
-\frac{p}{q}=(q-1) \frac{p}{q}-p \in M
$$

Now, if $\theta \in M$ is irrational, then $D \equiv\{n \theta+m: n, m \in \mathbb{Z}, n>0\} \subseteq M$. Since $D$ is dense in $\mathbb{R}$ and $M$ is closed, then $M=\mathbb{R}$ and so $-\theta$ is in $M$.

Since the only closed additive subgroups of $\mathbb{R}$ containing $\mathbb{Z}$ are $\frac{1}{n} \mathbb{Z}$ for some positive integer $n$ or the entire group $\mathbb{R}$, we have the following.

Proposition 3.2. Let $S$ be a SIS. Then either $S$ is translation-invariant, or there exists a maximum positive integer $n$ such that $S$ is $\frac{1}{n} \mathbb{Z}$-invariant. 
Proposition 3.2 suggests the following definition.

Definition 3.3. Given a shift-invariant space $S$, we say that $S$ has invariance order $n$ if $n$ is the maximum positive integer such that $S$ is $\frac{1}{n} \mathbb{Z}$-invariant. If this maximum does not exist, we say that $S$ has invariance order $\infty$; in this case $S$ is translation-invariant.

Remark 3.4. Note that the invariance order of any SIS is at least 1 , since $S$ is $\mathbb{Z}$ invariant. Also, if $S$ has invariance order $n$, then $S$ is not invariant under translation by any real number $y$ in the range $0<y<1 / n$. Furthermore, if $y \geq 1 / n, S$ can only be invariant under translation by $y$ if $y$ is a multiple of $1 / d$ where $d$ divides $n$. In particular, if the order of invariance of $S$ is a prime number $p$, there exist no other integers $m>1$ such that $S$ is invariant under translations by $1 / m$.

\section{Characterization of $\frac{1}{n}$-Invariance}

In this part we will characterize those shift-invariant spaces that are $\frac{1}{n} \mathbb{Z}$-invariant.

4.1. Notation. We will use the following notation throughout the remainder of this paper.

Given a fixed positive integer $n$, we partition the real line into $n$ sets, each of which is $n \mathbb{Z}$-periodic, as follows. For $k=0, \ldots, n-1$ define,

$$
B_{k}=\bigcup_{j \in \mathbb{Z}}([k, k+1)+n j) .
$$

Note that $B_{k}$ implicitly depends on the choice of $n$.

Given a SIS $S \subseteq L^{2}(\mathbb{R})$, we associate the following subspaces:

$$
U_{k}=\left\{f \in L^{2}(\mathbb{R}): \widehat{f}=\widehat{g} \chi_{B_{k}} \text { for some } g \in S\right\}, \quad k=0, \ldots, n-1 .
$$

The spaces $U_{k}$ are mutually orthogonal since the sets $B_{k}$ are disjoint (up to sets of measure zero).

If $f \in S$ and $0 \leq k \leq n-1$, then we let $f^{k}$ denote the function defined by

$$
\widehat{f^{k}}=\widehat{f} \chi_{B_{k}} .
$$

Letting $P_{k}$ denote the orthogonal projection onto $\left\{f: \operatorname{supp}(\hat{f}) \subseteq B_{k}\right\}$, we have that

$$
U_{k}=P_{k}(S) \text { and } f^{k}=P_{k} f .
$$

Note that integer translations commute with the projections $P_{k}$ : if $j \in \mathbb{Z}$ and $k=0, \ldots, n-1$, then

$$
T_{j} P_{k}=P_{k} T_{j} .
$$


4.2. Preliminary results. We will need the following result from dBVR94a].

Proposition 4.1 ([BBVR94a $)$. Let $f \in L^{2}(\mathbb{R})$ be given. If $g \in \mathfrak{S}(f)$, then there exists a $\mathbb{Z}$-periodic function $m$ such that $\widehat{g}=m \widehat{f}$.

Conversely, if $m$ is a $\mathbb{Z}$-periodic function such that $m \widehat{f} \in L^{2}(\mathbb{R})$, then the function $g$ defined by $\widehat{g}=m \widehat{f}$ belongs to $\mathfrak{S}(f)$.

We will also need a version of the preceding result for spaces that are $\frac{1}{n} \mathbb{Z}$-invariant instead of shift-invariant. This follows easily by rescaling.

Corollary 4.2. Let $f \in L^{2}(\mathbb{R})$ and $n \in \mathbb{N}$ be given, and set

$$
\mathfrak{S}\left(f, \frac{1}{n} \mathbb{Z}\right)=\overline{\operatorname{span}}\left\{T_{j / n} f: j \in \mathbb{Z}\right\} .
$$

If $g \in \mathfrak{S}\left(f, \frac{1}{n} \mathbb{Z}\right)$, then there exists a $n \mathbb{Z}$-periodic function $m$ such that $\widehat{g}=m \widehat{f}$.

Conversely, if $m$ is an $n \mathbb{Z}$-periodic function such that $m \widehat{f} \in L^{2}(\mathbb{R})$, then the function $g$ defined by $\widehat{g}=m \widehat{f}$ belongs to $\mathfrak{S}\left(f, \frac{1}{n} \mathbb{Z}\right)$.

4.3. Characterization of $\frac{1}{n}$-invariance in terms of subspaces. The periodicity of the $B_{k}$ sets yields the following lemma.

Lemma 4.3. Let $S$ be a SIS. Assume that the subspace $U_{k} \subseteq S$. Then for each $k=0, \ldots, n-1, U_{k}$ is a SIS that is also $\frac{1}{n} \mathbb{Z}$-invariant.

Proof. Fix $0 \leq k \leq n-1$, and choose any $f \in U_{k}$. There exists a $g \in S$ such that $\widehat{f}=\widehat{g} \chi_{B_{k}}$. Since $S$ is shift-invariant and $g \in S$, we have that $e^{-2 \pi i s \omega} \widehat{g}(\omega)$ is in $\widehat{S}$, for all $s \in \mathbb{Z}$. Hence

$$
e^{-2 \pi i s \omega} \widehat{f}(\omega)=e^{-2 \pi i s \omega} \widehat{g}(\omega) \chi_{B_{k}}(\omega) \in \widehat{U}_{k} .
$$

Therefore $T_{s} f \in U_{k}$, so $U_{k}$ is invariant under integer translates.

Suppose now that $f_{j} \in U_{k}$ and $f_{j} \rightarrow f$ in $L^{2}(\mathbb{R})$. Since $U_{k} \subseteq S$ and $S$ is closed, $f$ must be in $S$. Further,

$$
\left\|\widehat{f}_{j}-\widehat{f}\right\|_{2}^{2}=\left\|\left(\widehat{f}_{j}-\widehat{f}\right) \chi_{B_{k}}\right\|_{2}^{2}+\left\|\left(\widehat{f}_{j}-\widehat{f}\right) \chi_{B_{k}^{\mathrm{C}}}\right\|_{2}^{2}=\left\|\widehat{f}_{j}-\widehat{f} \chi_{B_{k}}\right\|_{2}^{2}+\left\|\widehat{f} \chi_{B_{k}^{\mathrm{C}}}\right\|_{2}^{2} .
$$

Since the left-hand side converges to zero, we must have that $\widehat{f} \chi_{B_{k}^{\mathrm{C}}}=0$ a.e., and that $\widehat{f}_{j} \rightarrow \widehat{f} \chi_{B_{k}}$ in $L^{2}(\mathbb{R})$. Since we also have $\widehat{f}_{j} \rightarrow \widehat{f}$, we conclude that

$$
\widehat{f}=\widehat{f} \chi_{B_{k}} \text { a.e. }
$$

Consequently $f \in U_{k}$, so $U_{k}$ is closed.

Finally, to see that $U_{k}$ is $\frac{1}{n} \mathbb{Z}$-invariant, define

$$
h(\omega)=e^{-\frac{2 \pi i \omega}{n}} \sum_{j=-k}^{n-1-k} e^{\frac{2 \pi i j}{n}} \chi_{B_{k+j}}(\omega) .
$$

Note that $|h(\omega)|=1$ and that $h$ is $\mathbb{Z}$-periodic. Furthermore, if $\omega \in B_{k}$ and $-k \leq$ $j \leq n-1-k$, then $\chi_{B_{k+j}}(\omega)$ can be nonzero only when $j=0$. Hence:

$$
\omega \in B_{k} \quad \Longrightarrow \quad h(\omega)=e^{-\frac{2 \pi i \omega}{n}} \text {. }
$$


If $f \in U_{k}$ then, $\operatorname{since} \operatorname{supp}(\hat{f}) \subseteq B_{k}$, we have

$$
e^{-\frac{2 \pi i \omega}{n}} \widehat{f}(\omega)=h(\omega) \widehat{f}(\omega)
$$

However, since $U_{k}$ is $\mathbb{Z}$-invariant, we have by Proposition 4.1 that $h \widehat{f} \in \widehat{U_{k}}$. Therefore $e^{-\frac{2 \pi i \omega}{n}} \widehat{f}(\omega) \in \widehat{U_{k}}$, which implies that $T_{1 / n} f \in U_{k}$.

This leads to the following characterization.

Theorem 4.4. If $S \subseteq L^{2}(\mathbb{R})$ is a SIS, then the following are equivalent.

(a) $S$ is $\frac{1}{n} \mathbb{Z}$-invariant.

(b) $U_{k} \subseteq S$ for $k=0, \ldots, n-1$.

(c) If $f \in S$, then $f^{k}=P_{k} f \in S$ for each $k=0, \ldots, n-1$.

Moreover, in case these hold we have that $S$ is the orthogonal direct sum

$$
S=U_{0} \dot{\oplus} \ldots \dot{\oplus} U_{n-1},
$$

with each $U_{k}$ being a (possibly trivial) $\frac{1}{n} \mathbb{Z}$-invariant SIS.

Proof. (a) $\Rightarrow$ (b). Assume that $S$ is $\frac{1}{n} \mathbb{Z}$-invariant and fix $0 \leq k \leq n-1$ and $f \in U_{k}$. By definition of $U_{k}$, we have that $\widehat{f}=\widehat{g} \chi_{B_{k}}$ for some $g \in S$. Since $\chi_{B_{k}}$ is $n \mathbb{Z}$-periodic and bounded, Corollary 4.2 implies that $f \in \mathfrak{S}\left(g, \frac{1}{n} \mathbb{Z}\right) \subseteq S$.

(b) $\Rightarrow$ (a). Suppose that $U_{k} \subseteq S$ for each $k=0, \ldots, n-1$.

Note that Lemma 4.3 implies that $U_{k}$ is $\frac{1}{n} \mathbb{Z}$-invariant, and we also have that the $U_{k}$ are mutually orthogonal since the sets $B_{k}$ are disjoint.

Suppose that $f \in S$. Then $f=f^{0}+\cdots+f^{n-1}$ where $\widehat{f^{k}}=\widehat{f} \chi_{B_{k}}$. This implies that $f \in U_{0} \dot{\oplus} \ldots \dot{\oplus} U_{n-1}$, and consequently $S$ is the orthogonal direct sum

$$
S=U_{0} \dot{\oplus} \ldots \dot{\oplus} U_{n-1}
$$

As each $U_{k}$ is $\frac{1}{n} \mathbb{Z}$-invariant, it follows that $S$ is $\frac{1}{n} \mathbb{Z}$-invariant as well.

(b) $\Leftrightarrow(\mathrm{c})$. This is a restatement of the definition of $U_{k}$.

Corollary 4.5. Let $S$ be a SIS. If there exists a $k \in\{0, \ldots, n-1\}$ such that $\operatorname{supp}(\widehat{f}) \subseteq B_{k}$ for all $f \in S$, then $S$ is $\frac{1}{n} \mathbb{Z}$-invariant.

Remark 4.6. It is interesting to note that the subspaces $U_{k}$ satisfy:

$$
U_{k}=P_{k}(S)=\left\{f \in L^{2}(\mathbb{R}): \operatorname{supp}(\hat{f}) \subseteq B_{k}\right\} \cap S .
$$

That is, the projections and the restrictions of $S$ yield valid tests for $\frac{1}{n} \mathbb{Z}$-invariance. 
4.4. Characterization of $\frac{1}{n}$-invariance in terms of generators. We will show now that the conditions for $\frac{1}{n} \mathbb{Z}$-invariance can be formulated in terms of properties of a set of generators of the SIS.

Theorem 4.7. Let $\mathcal{F}$ be a set of generators for a $S I S S$, i.e., $S=\mathfrak{S}(\mathcal{F})$. Then the following statements are equivalent.

(a) $S$ is $\frac{1}{n} \mathbb{Z}$-invariant.

(b) $P_{k} \mathcal{F}=\left\{f^{k}: f \in \mathcal{F}\right\} \subseteq S$ for $k=0, \ldots, n-1$.

Proof. (a) $\Rightarrow$ (b). This is a consequence of Theorem 4.4.

(b) $\Rightarrow$ (a). Suppose that statement (b) holds. Then, by hypothesis, $V_{k}=$ $\mathfrak{S}\left(P_{k} \mathcal{F}\right) \subseteq S$, and, by Corollary 4.5, $V_{k}$ is $\frac{1}{n} \mathbb{Z}$-invariant. Furthermore, $V_{j} \perp V_{k}$ when $j \neq k$. If $f \in \mathcal{F}$, then $f=f^{0}+\cdots+f^{n-1} \in V_{1} \dot{\oplus} \cdots \dot{\oplus} V_{n-1}$. Consequently, $S=\mathfrak{S}(\mathcal{F})=V_{1} \dot{\oplus} \cdots \dot{\oplus} V_{n-1}$. As each $V_{k}$ is $\frac{1}{n} \mathbb{Z}$-invariant, it follows that $S$ is as well.

It is known that it is always possible to choose a (possibly infinite) set of generators of a SIS in such a way that the integer translates of the generators actually forms a frame for the SIS (see Theorem 4.16). This is particularly important in applications, and we examine this situation next.

Recall that a countable collection of vectors $\left\{v_{\alpha}: \alpha \in \Lambda\right\}$ forms a frame for a Hilbert space $H$ if there exist constants $A, B$ (called frame bounds) such that

$$
\forall w \in H, \quad A\|w\|^{2} \leq \sum_{\alpha \in \Lambda}\left|\left\langle w, v_{\alpha}\right\rangle\right|^{2} \leq B\|w\|^{2} .
$$

If we can take $A=B=1$, then the frame is called a Parseval frame.

The next result shows that if the integer translates of the generators of a SIS form a frame, then the set of integer translations of the "cutoffs" of the generators remains a frame.

Theorem 4.8. Assume that $S$ is a SIS that is $\frac{1}{n} \mathbb{Z}$-invariant, and that $\mathcal{F} \subseteq S$ is such that $\mathcal{T}_{\mathbb{Z}}(\mathcal{F})$ is a frame for $S$ with frame bounds $A, B$. Then

$$
\mathcal{T}_{\mathbb{Z}}\left(P_{k} \mathcal{F}\right)=\left\{T_{j} f^{k}: f \in \mathcal{F}, j \in \mathbb{Z}\right\}
$$

is a frame for $U_{k}=\mathfrak{S}\left(P_{k} \mathcal{F}\right)$ with frame bounds $A, B$. Further,

$$
\mathcal{T}_{\mathbb{Z}}\left(\bigcup_{k=0}^{n-1} P_{k} \mathcal{F}\right)=\left\{T_{j} f^{k}: f \in \mathcal{F}, j \in \mathbb{Z}, k=0, \ldots, n-1\right\}
$$

is a frame for $S$ with frame bounds $A, B$.

Proof. By hypothesis,

$$
\forall g \in S, \quad A\|g\|_{2}^{2} \leq \sum_{j \in \mathbb{Z}} \sum_{f \in \mathcal{F}}\left|\left\langle g, T_{j} f\right\rangle\right|^{2} \leq B\|g\|_{2}^{2} .
$$


Suppose that $g \in U_{k}$. Then since $P_{k}$ commutes with integer translations, we have

$$
\begin{aligned}
\sum_{j \in \mathbb{Z}} \sum_{f \in \mathcal{F}}\left|\left\langle g, T_{j} P_{k} f\right\rangle\right|^{2} & =\sum_{j \in \mathbb{Z}} \sum_{f \in \mathcal{F}}\left|\left\langle g, P_{k} T_{j} f\right\rangle\right|^{2} \\
& =\sum_{j \in \mathbb{Z}} \sum_{f \in \mathcal{F}}\left|\left\langle P_{k} g, T_{j} f\right\rangle\right|^{2} \\
& =\sum_{j \in \mathbb{Z}} \sum_{f \in \mathcal{F}}\left|\left\langle g, T_{j} f\right\rangle\right|^{2} .
\end{aligned}
$$

Combining this with (3) , we see that $\mathcal{T}_{\mathbb{Z}}\left(P_{k} \mathcal{F}\right)$ is a frame for $U_{k}$ with frame bounds $A, B$.

Suppose now that $g \in S$. Then since $S$ is the orthogonal direct sum of the $U_{k}$, we have that

$$
\sum_{j \in \mathbb{Z}} \sum_{f \in \mathcal{F}} \sum_{k=0}^{n-1}\left|\left\langle g, T_{j} P_{k} f\right\rangle\right|^{2}=\sum_{k=0}^{n-1} \sum_{j \in \mathbb{Z}} \sum_{f \in \mathcal{F}}\left|\left\langle P_{k} g, T_{j} f\right\rangle\right|^{2} \leq B \sum_{k=0}^{n-1}\left\|P_{k} g\right\|_{2}^{2}=B\|g\|_{2}^{2} .
$$

The estimate from below is similar, so we see that $\mathcal{T}_{\mathbb{Z}}\left(\bigcup_{k=0}^{n-1} P_{k} \mathcal{F}\right)$ is a frame for $S$, with frame bounds $A, B$.

4.5. Characterization of $\frac{1}{n}$-invariance in terms of fibers. A useful tool in the theory of shift-invariant spaces is based on early work of Helson Hel64. An $L^{2}(\mathbb{R})$ function is decomposed into "fibers." This produces a characterization of SIS in terms of closed subspaces of $\ell^{2}(\mathbb{Z})$ (the fiber spaces). For a detailed description of this approach, see [Bow00] and the references therein.

Definition 4.9. Given $f \in L^{2}(\mathbb{R})$ and $\omega \in[0,1)$, the fiber $\widehat{f}_{\omega}$ of $f$ at $\omega$ is the sequence

$$
\widehat{f}_{\omega}=\{\widehat{f}(\omega+k)\}_{k \in \mathbb{Z}} .
$$

If $f$ is in $L^{2}(\mathbb{R})$, then the fiber $\widehat{f}_{\omega}$ belongs to $\ell^{2}(\mathbb{Z})$ for almost every $\omega \in[0,1)$.

Definition 4.10. Given a subspace $V$ of $L^{2}(\mathbb{R})$ and $\omega \in[0,1)$, the fiber space of $V$ at $\omega$ is

$$
\mathcal{J}_{V}(\omega)=\overline{\left\{\widehat{f}_{\omega}: f \in V \text { and } \widehat{f}_{\omega} \in \ell^{2}(\mathbb{Z})\right\}}
$$

where the closure is taken in the norm of $\ell^{2}(\mathbb{Z})$.

The map assigning to each $\omega$ the fiber space $\mathcal{J}_{V}(\omega)$ is known in the literature as the range function of $V$.

For a proof that, for almost every $\omega, \mathcal{J}_{V}(\omega)$ is a well-defined closed subspace of $\ell_{2}(\mathbb{Z})$ and that shift-invariant spaces can be characterized through range functions, see Bow00, Hel64].

We will need the following two results.

Proposition 4.11 ([Hel64]). If $S$ is a SIS, then

$$
S=\left\{f \in L^{2}(\mathbb{R}): \widehat{f}_{\omega} \in \mathcal{J}_{S}(\omega) \text { for a.e. } \omega\right\} .
$$


Proposition 4.12. Let $S_{1}$ and $S_{2}$ be SISs. If $S=S_{1} \dot{\oplus} S_{2}$, then

$$
\mathcal{J}_{S}(\omega)=\mathcal{J}_{S_{1}}(\omega) \dot{\oplus} \mathcal{J}_{S_{2}}(\omega), \quad \text { a.e. } \omega .
$$

The converse of Proposition 4.12 is also true, but will not be needed.

Combining Theorem 4.4 with Proposition 4.11 yields the following characterization of $\frac{1}{n}$-invariance in terms of the fiber spaces.

Theorem 4.13. Let $S$ be a SIS. Then the following statements are equivalent.

(a) $S$ is $\frac{1}{n} \mathbb{Z}$-invariant.

(b) $\mathcal{J}_{U_{k}}(\omega) \subseteq \mathcal{J}_{S}(\omega)$ for almost every $\omega$ and each $k=0, \ldots, n-1$.

For the finitely generated case we can obtain a slightly simpler characterization of $\frac{1}{n} \mathbb{Z}$-invariance.

Theorem 4.14. If $S$ is a finitely generated SIS, then the following statements are equivalent.

(a) $S$ is $\frac{1}{n} \mathbb{Z}$-invariant.

(b) For almost every $\omega \in[0,1)$,

$$
\operatorname{dim}\left(\mathcal{J}_{S}(\omega)\right)=\sum_{k=0}^{n-1} \operatorname{dim}\left(\mathcal{J}_{U_{k}}(\omega)\right)
$$

Proof. (a) $\Rightarrow$ (b). If $S$ is $\frac{1}{n} \mathbb{Z}$-invariant then $S=\dot{\oplus}_{k=0}^{n-1} U_{k}$. This is an orthogonal direct sum, so Proposition 4.12 implies that $\mathcal{J}_{S}(\omega)=\dot{\oplus}_{k=0}^{n-1} \mathcal{J}_{U_{k}}(\omega)$ for a.e. $\omega$, with this sum also orthogonal. The equality of dimensions in statement (b) therefore holds.

(b) $\Rightarrow$ (a). Suppose that statement (b) holds. It is clear that the inclusion $\mathcal{J}_{S}(\omega) \subseteq \dot{\oplus}_{k=0}^{n-1} \mathcal{J}_{U_{k}}(\omega)$ holds for a.e. $\omega$. Since the spaces $U_{k}$ are orthogonal, Proposition 4.12 implies that, for a.e. $\omega$, the spaces $\mathcal{J}_{U_{k}}(\omega)$ are also orthogonal. Counting dimensions and applying statement (b), we conclude that $\mathcal{J}_{S}(\omega)=\dot{\oplus}_{k=0}^{n-1} \mathcal{J}_{U_{k}}(\omega)$ for a.e. $\omega$.

Suppose now that $f \in U_{k}$. Then $\widehat{f}_{\omega} \in \mathcal{J}_{U_{k}}(\omega) \subseteq \mathcal{J}_{S}(\omega)$ for a.e. $\omega$, so Proposition 4.11 implies that $f \in S$. Thus $U_{k} \subseteq S$ for each $k$, so it follows from Theorem 4.4 that $S$ is $\frac{1}{n} \mathbb{Z}$-invariant.

Remark 4.15. Given a SIS $V$, the function $D_{V}(\omega) \equiv \operatorname{dim}\left(\mathcal{J}_{V}(\omega)\right)$ defined for $\omega \in$ $[0,1)$ is known in the literature as the Dimension function or Multiplicity function of the shift-invariant space $V$. So, condition (b) of Theorem 4.14 is a statements about dimension functions of the shift invariant spaces involved.

4.6. The Bownik decomposition and $\frac{1}{n}$-invariance. In Bow00, Bownik obtained a decomposition for general shift-invariant spaces, extending the earlier works dBVR94a and dBDR94b, which applied to the finitely generated case. We will apply this decomposition to shift-invariant spaces that are $\frac{1}{n} \mathbb{Z}$-invariant. 
Theorem 4.16 (Bownik). Let $S \subseteq L^{2}(\mathbb{R})$ be a SIS. Then for each $j \in \mathbb{N}$ we can find a function $\varphi_{j} \in L^{2}(\mathbb{R})$ such that $\mathcal{T}_{\mathbb{Z}}\left(\varphi_{j}\right)$ is a Parseval frame for $\mathfrak{S}\left(\varphi_{j}\right)$, and furthermore

$$
S=\bigoplus_{j \in \mathbb{N}} \mathfrak{S}\left(\varphi_{j}\right)
$$

Note that a consequence of this theorem is that every SIS always has a set of generators whose integer translates form a Parseval frame of the SIS.

By applying Theorem 4.16 to each space $U_{k}$, we obtain the following result.

Theorem 4.17. Let $S$ be a SIS that is $\frac{1}{n} \mathbb{Z}$-invariant. Then there exist functions $\varphi_{k, j} \in L^{2}(\mathbb{R})$ such that

$$
S=\bigoplus_{k=0}^{n-1} \bigoplus_{j \in \mathbb{N}} \mathfrak{S}\left(\varphi_{k, j}\right),
$$

with the following properties holding.

(a) $\mathcal{T}_{\mathbb{Z}}\left(\varphi_{k, j}\right)$ is a Parseval frame for $\mathfrak{S}\left(\varphi_{k, j}\right)$.

(b) $\mathfrak{S}\left(\varphi_{k, j}\right) \subseteq U_{k}$ for each $j \in \mathbb{N}$, and

$$
U_{k}=\bigoplus_{j \in \mathbb{N}} \mathfrak{S}\left(\varphi_{k, j}\right) .
$$

(c) Each space $\mathfrak{S}\left(\varphi_{k, j}\right)$ is $\frac{1}{n} \mathbb{Z}$-invariant.

\section{Finitely Generated Shift-Invariant Spaces And $\frac{1}{n}$-Invariance}

In this section we will apply some of the general results obtained so far to the particular case of finitely generated shift-invariant spaces. We will use the concept of the Gramian. This is a common tool in the study of finitely generated shift-invariant spaces; see for example dBDR94b, [RS95, ACM07.

\subsection{Characterization of $\frac{1}{n}$-invariance in terms of the Gramian.}

Definition 5.1. Let $\Phi=\left\{\varphi_{1}, \ldots, \varphi_{m}\right\}$ be a collection of finitely many functions in $L^{2}(\mathbb{R})$. Then the Gramian $G_{\Phi}$ of $\Phi$ is the $m \times m$ matrix of $\mathbb{Z}$-periodic functions

$$
\left[G_{\Phi}(\omega)\right]_{i j}=\left\langle\left(\widehat{\varphi}_{i}\right)_{\omega},\left(\widehat{\varphi}_{j}\right)_{\omega}\right\rangle=\sum_{k \in \mathbb{Z}} \widehat{\varphi}_{i}(\omega+k) \overline{\widehat{\varphi}_{j}(\omega+k)}, \quad \omega \in \mathbb{R},
$$

where $\left(\widehat{\varphi}_{j}\right)_{\omega}$ is the fiber of $\varphi_{j}$ at $\omega$.

We consider now the SIS $S=\mathfrak{S}(\Phi)$ generated by the set $\Phi=\left\{\varphi_{1}, \ldots, \varphi_{m}\right\}$. It is known dBDR94b] that if $f \in \mathfrak{S}(\Phi)$, then there exist $\mathbb{Z}$-periodic functions $a_{1}, \ldots, a_{m}$ such that

$$
\widehat{f}(\omega)=\sum_{j=1}^{m} a_{j}(\omega) \widehat{\varphi}_{j}(\omega), \quad \text { a.e. } \omega \text {. }
$$


This implies that the fiber spaces $\mathcal{J}_{S}(\omega)$ are generated by the fibers of the generators of $S$ at $\omega$ (see also Bow00]). That is, for almost every $\omega$ we have that

$$
\mathcal{J}_{S}(\omega)=\operatorname{span}\left\{\left(\widehat{\varphi}_{j}\right)_{\omega}: j=1, \ldots, m\right\} .
$$

Therefore

$$
\operatorname{dim}\left(\mathcal{J}_{S}(\omega)\right)=\operatorname{rank}\left[G_{\Phi}(\omega)\right]
$$

for almost every $\omega$.

In the same way, since the SIS $U_{k}$ is generated by $\Phi^{k}=P_{k} \Phi=\left\{\varphi_{1}^{k}, \ldots, \varphi_{m}^{k}\right\}$, where $\varphi_{j}^{k}=P_{k} \varphi_{j}$, we have for almost every $\omega$ that the fiber spaces $\mathcal{J}_{U_{k}}(\omega)$ satisfy

$$
\mathcal{J}_{U_{k}}(\omega)=\operatorname{span}\left\{\left(\widehat{\varphi_{j}^{k}}\right)_{\omega}: j=1, \ldots, m\right\} .
$$

Let us denote by $G_{\Phi^{k}}$ the Gramian matrix associated with the generators of $U_{k}$. Then, as above we have that $\operatorname{dim}\left(\mathcal{J}_{U_{k}}(\omega)\right)=\operatorname{rank}\left[G_{\Phi^{k}}(\omega)\right]$ for almost every $\omega$ and $k=0, \ldots, n-1$. Now Theorem 4.14 can be restated in the following way.

Theorem 5.2. If $S=\mathfrak{S}(\Phi)$ is the SIS generated by $\Phi=\left\{\varphi_{1}, \ldots, \varphi_{m}\right\}$, then the following statements are equivalent.

(a) $S$ is $\frac{1}{n} \mathbb{Z}$-invariant.

(b) For almost every $\omega \in[0,1)$ we have

$$
\operatorname{rank}\left[G_{\Phi}(\omega)\right]=\sum_{k=0}^{n-1} \operatorname{rank}\left[G_{\Phi^{k}}(\omega)\right] .
$$

5.2. Implications for frequency support. As a consequence of Theorem 5.2 we deduce an interesting result about the supports of the Fourier transforms of the generators of a SIS.

Theorem 5.3. Let $S=\mathfrak{S}(\Phi)$ be the SIS generated by $\Phi=\left\{\varphi_{1}, \ldots, \varphi_{m}\right\}$, and define

$$
E_{j}=\left\{\omega \in[0,1): \operatorname{rank}\left[G_{\Phi}(\omega)\right]=j\right\}, \quad j=0, \ldots, m .
$$

If $S$ is $\frac{1}{n} \mathbb{Z}$-invariant, then for each interval $I \subseteq \mathbb{R}$ of length $n$, we have that for each $h=1, \ldots, m$

$$
\left|\left\{\omega \in I: \widehat{\varphi_{h}}(\omega)=0\right\}\right| \geq \sum_{j=0}^{n-1}(n-j)\left|E_{j}\right| .
$$

In particular if $n>m$ we have,

$$
\left|\left\{\omega \in I: \widehat{\varphi_{h}}(\omega)=0\right\}\right| \geq n-m .
$$

Proof. The measurability of the sets $E_{j}$ follows from the results of Helson Hel64, e.g., see BK06] for an argument of this type.

It is enough to prove the theorem for the interval $I=[0, n)$.

We note that the set

$$
K_{1}=\left\{\omega \in[0,1):\left(\widehat{\varphi_{h}}\right)_{\omega} \in \ell^{2}(\mathbb{Z}) \text { for } h=1, \ldots, m\right\}
$$


has full measure. Therefore

$$
K_{n}=\bigcup_{k=0}^{n-1}\left(K_{1}+k\right)
$$

is a subset of $[0, n)$ of measure $n$.

Fix any particular $j \in\{0, \ldots, m\}$. By Theorem 5.2 ,

$$
\operatorname{rank}\left[G_{\Phi}(\omega)\right]=\sum_{k=0}^{n-1} \operatorname{rank}\left[G_{\Phi^{k}}(\omega)\right], \quad \text { a.e. } \omega
$$

Therefore, for $j<n$, if $\omega \in E_{j}$ then at least $n-j$ terms on the right-hand side of equation (5) must vanish. That is, given such an $\omega$, there exists a subset of $\{0, \ldots, n-1\}$ with at least $n-j$ elements such that for each $k$ in this subset

$$
\operatorname{rank}\left[G_{\Phi^{k}}(\omega)\right]=0
$$

In particular, for each $h \in\{1, \ldots, m\}$ we have that, for $j<n$ and $\omega \in E_{j}$,

$$
\#\left\{k \in\{0, \ldots, n-1\}: \widehat{\varphi_{h}}(\omega+k)=0\right\} \geq n-j .
$$

On the other hand, using that the sets $E_{j}$ are disjoint, we have

$$
\begin{aligned}
& \left\{\omega \in K_{n}: \widehat{\varphi_{h}}(\omega)=0\right\} \\
& \quad=\left\{\omega+k: \omega \in K_{1}, 0 \leq k \leq n-1 \text { and } \widehat{\varphi_{h}}(\omega+k)=0\right\} \\
& \quad=\bigcup_{j=0}^{m}\left\{\omega+k: \omega \in K_{1} \cap E_{j}, 0 \leq k \leq n-1 \text { and } \widehat{\varphi_{h}}(\omega+k)=0\right\} .
\end{aligned}
$$

Consequently, from (6) and the last equation it follows that,

$$
\begin{aligned}
& \left|\left\{\omega \in K_{n}: \widehat{\varphi_{h}}(\omega)=0\right\}\right| \\
& \quad=\sum_{j=0}^{m} \mid\left\{\omega+k: \omega \in K_{1} \cap E_{j}, 0 \leq k \leq n-1 \text { and } \widehat{\varphi_{h}}(\omega+k)=0\right\} \mid \\
& \quad=\sum_{j=0}^{m} \int_{E_{j}} \#\left\{k \in\{0, \ldots, n-1\}: \widehat{\varphi_{h}}(\omega+k)=0\right\} d \omega \\
& \quad \geq \sum_{j=0}^{n-1}(n-j)\left|E_{j}\right| .
\end{aligned}
$$

Furthermore, if $n>m$,

$$
\sum_{j=0}^{m}(n-j)\left|E_{j}\right| \geq \sum_{j=0}^{m}(n-m)\left|E_{j}\right|=(n-m) .
$$


The measurability of the function $\omega \longmapsto \#\left\{k \in\{0, \ldots, n-1\}: \widehat{\varphi_{h}}(\omega+k)=0\right\}$, follows from the fact that

$$
\begin{aligned}
& \left\{w \in E_{j}: \#\left\{k \in\{0, \ldots, n-1\}: \widehat{\varphi_{h}}(\omega+k)=0\right\} \geq s\right\} \\
= & \bigcup_{0 \leq k_{1}<\cdots<k_{s}<n} \bigcap_{i=1}^{s}\left\{\omega \in E_{j}: \widehat{\varphi_{h}}\left(\omega+k_{i}\right)=0\right\} .
\end{aligned}
$$

Note that if $S$ is a principal SIS, say $S=\mathfrak{S}(\varphi)$, then the Gramian is scalar-valued; since

$$
G_{\varphi}(\omega)=\left\langle\widehat{\varphi}_{\omega}, \widehat{\varphi}_{\omega}\right\rangle=\sum_{k \in \mathbb{Z}}|\widehat{\varphi}(\omega+k)|^{2} .
$$

Applying Theorem 5.3 to this case, we obtain the following corollary.

Corollary 5.4. Let $\varphi \in L^{2}(\mathbb{R})$ be given. If the $S I S \mathfrak{S}(\varphi)$ is $\frac{1}{n} \mathbb{Z}$-invariant for some $n>1$, then $\widehat{\varphi}$ must vanish on a set of infinite Lebesgue measure. Furthermore, for each interval $I \subseteq \mathbb{R}$ of length $n$, we have that

$$
|\{\omega \in I: \widehat{\varphi}(\omega)=0\}| \geq n\left|E_{0}\right|+(n-1)\left|E_{1}\right| \geq n-1,
$$

where $E_{0}=\left\{\omega \in[0,1): G_{\varphi}(\omega)=0\right\}$ and $E_{1}=\left\{\omega \in[0,1): G_{\varphi}(\omega) \neq 0\right\}$

This yields the following fact regarding the order of invariance of a principal SIS generated by a compactly supported function.

Proposition 5.5. If a nonzero function $\varphi \in L^{2}(\mathbb{R})$ has compact support, then $\mathfrak{S}(\varphi)$ has invariance order one. That is $\mathfrak{S}(\varphi)$ is not $\frac{1}{n} \mathbb{Z}$-invariant for any $n>1$.

Proof. Because of Corollary 5.4, if $\mathfrak{S}(\varphi)$ is $\frac{1}{n} \mathbb{Z}$-invariant with $n>1$, then $\widehat{\varphi}$ must vanish on a set of positive measure. Since $\varphi$ has compact support, the Paley-Wiener Theorem implies that $\varphi=0$ a.e.

It is not difficult to construct a function $\varphi \in L^{2}(\mathbb{R})$ such that $\widehat{\varphi}$ is compactly supported in frequency yet the SIS $\mathfrak{S}(\varphi)$ is not translation-invariant. In fact, we have the following consequence of Corollary 5.4 .

Corollary 5.6. If $\varphi \in L^{2}(\mathbb{R})$ and $\mathfrak{S}(\varphi)$ is translation-invariant, then $|\operatorname{supp}(\widehat{\varphi})| \leq 1$.

Remark 5.7. As one of the referees pointed out, Proposition [5.5 is known and follows readily from Proposition 4.1,

Likewise, Corollary [5.6 can be obtained from properties of the dimension function of a SIS. For this, observe that since $\mathfrak{S}(\varphi)$ is translation invariant then,

$$
D_{\mathfrak{S}(\varphi)}=\sum_{k \in \mathbb{Z}} \chi_{\operatorname{supp}(\hat{\varphi})}(\omega+k) .
$$

Now using that $\mathfrak{S}(\varphi)$ is principal, we have $D_{\mathfrak{S}(\varphi)} \leq 1$. Thus integrating both sides over $[0,1]$, yields $|\operatorname{supp}(\widehat{\varphi})| \leq 1$. For properties of the dimension function of a SIS, see for example [BM99] or [BR03]. 
Remark 5.8. Assume now that $\varphi \in L^{2}(\mathbb{R})$ and $\mathfrak{S}(\varphi)$ is translation-invariant. Using the argument in Remark 5.7 we have that $\sum_{k \in \mathbb{Z}} \chi_{\operatorname{supp}(\hat{\varphi})}(\omega+k) \leq 1$. This implies that $\operatorname{supp}(\hat{\varphi})$ is a subset of a set of representatives of the quotient $\mathbb{R} / \mathbb{Z}$. That is, $\operatorname{supp}(\hat{\varphi})$ is a subset of a tile of $\mathbb{R}$, what is a refinement of Corollary 5.6. Translationinvariance of multiresolution analyses is connected to tilings of $\mathbb{R}$. See for example Mad92.

5.3. Application to multiresolution analyses. For definitions and details on wavelets and multiresolution analyses, see Dau92 or Mal89.

Suppose that $\left\{V_{j}\right\}_{j \in \mathbb{Z}}$ is a multiresolution analysis (MRA) of $L^{2}(\mathbb{R})$. By definition, $V_{0}=\mathfrak{S}(\varphi)$ for some $\varphi \in L^{2}(\mathbb{R})$, called the scaling function, and $V_{j}$ is the image of $V_{0}$ under the unitary operator $D_{2^{j}} f(x)=2^{j / 2} f\left(2^{j} x\right)$.

The preceding results imply that if $\varphi$ is compactly supported (as is the case for the Daubechies scaling functions, for example), then the SIS $V_{0}$ has invariance order exactly 1 . Thus $V_{0}$ is invariant only under integer translations. The same remarks apply to the associated wavelet $\psi$ and wavelet SIS $W_{0}=\mathfrak{S}(\psi)$ if $\psi$ is compactly supported.

Further, if $\varphi$ is compactly supported, then at resolution level $j$, the subspace $V_{j}$ is invariant exactly under translations $2^{j} \mathbb{Z}$, and similarly for the wavelet space $W_{j}$ if $\psi$ is compactly supported. This includes all the spaces associated with the Daubechies scaling functions and wavelets, for example.

\section{Higher Dimensions}

The results of this article are for the line. The higher-dimensional case is much more involved due to the more complex structure of the closed additive subgroups of $\mathbb{R}^{d}$. This will be the subject of a forthcoming article.

\section{ACKNOWLEDGements}

We thank Magalí Anastasio for carefully reading the manuscript. We also thank the referees for very interesting observations that contributed to improvement of the paper and the argument in Remark 5.7.

\section{REFERENCES}

[ACM07] A. Aldroubi, C. Cabrelli, D. Hardin and U. Molter Optimal shift invariant spaces and their Parseval frame generators. Appl. Comput. Harmon. Anal., 23 (2007), no. 2, 273283.

[BM99] L. Baggett and K. D. Merrill Abstract harmonic analysis and wavelets in $\mathbf{R}^{n}$. The functional and harmonic analysis of wavelets and frames (San Antonio, TX, 1999), Contemp. Math.,247, 17-27, Amer. Math. Soc., Providence, RI, 1999.

[Bow00] M. Bownik, The structure of shift-invariant subspaces of $L^{2}\left(\mathbb{R}^{n}\right)$, J. Funct. Anal., 177(2) (2000), 282-309.

[BR03] M. Bownik and Z. Rzeszotnik The spectral function of shift-invariant spaces, Michigan Math. J. 51 (2003), no. 2, 387-414.

[BK06] M. Bownik and N. Kaiblinger, Minimal generator sets for finitely generated shiftinvariant subspaces of $L^{2}\left(\mathbb{R}^{n}\right)$, J. Math. Anal. Appl., 313(1) (2006), 342-352. 
[dBVR94a] C. de Boor, R. De Vore, and A. Ron, Approximation from shift-invariant subspaces of $L_{2}\left(\mathbf{R}^{d}\right)$, Trans. Amer. Math. Soc., 341(2) (1994), 787-806.

[dBDR94b] C. de Boor, R. De Vore, and A. Ron, The structure of finitely generated shift-invariant subspaces of $L_{2}\left(\mathbb{R}^{d}\right)$, J. Funct. Anal., 119(1) (1994), 37-78.

[CS03] C. K. Chui and Q. Sun, Tight frame oversampling and its equivalence to shift-invariance of affine frame operators, Proc. Amer. Math. Soc., 131(5) (2003), 1527-1538.

[Dau92] I. Daubechies, Ten Lectures on Wavelets, SIAM, Philadelphia, 1992.

[Grö01] K. Gröchenig, Foundations of Time-Frequency Analysis, Birkhäuser, Boston, 2001.

[Hel64] H. Helson, Lectures on Invariant Subspaces, Academic Press, New York-London, 1964.

[HW96] E. Hernández and G. Weiss, A First Course on Wavelets, CRC Press, Boca Raton, FL, 1996.

[HL01] J. A. Hogan and J. Lakey, Sampling and aliasing without translation-invariance, Proc. Fourth Int. Conf. on Sampling Theory and Applications (SampTA'01), Orlando, FL, 2001, 61-66.

[Mad92] W. R. Madich, Some elementary properties of multiresolution analyses of $L^{2}\left(\mathbb{R}^{n}\right)$, Wavelets, 259-294, Academic Press, Boston, 1992.

[Mal89] S. G. Mallat, Multiresolution approximations and wavelet orthonormal bases of $L^{2}(\mathbf{R})$, Trans. Amer. Math. Soc., 315(1) (1989), 69-87.

[Mal98] S. Mallat, A Wavelet Tour of Signal Processing, Academic Press, San Diego, CA, 1998.

[RS95] A. Ron and Z. Shen, Frames and stable bases for shift-invariant subspaces of $L^{2} \mathbb{R}^{d}$, Canad. J.Math. 47 (1995), 1051-1094.

[Web00] E. Weber, On the translation invariance of wavelet subspaces, J. Fourier Anal. Appl., 6(5) (2000), 551-558.

(A. Aldroubi) Department of Mathematics, Vanderbilt University, Nashville, TenNESSEE 37240-0001 USA

E-mail address: aldroubi@math.vanderbilt.edu

(C. Cabrelli) Departamento de Matemática, Facultad de Ciencias Exactas y Naturales, Universidad de Buenos Aires, Ciudad Universitaria, Pabellón I, 1428 Buenos Aires, Argentina and COniCEt, Consejo Nacional de Investigaciones Científicas y TÉCNICAS, Argentina

E-mail address: cabrelli@dm.uba.ar

(C. Heil) School of Mathematics, Georgia Institute of Technology, Atlanta, GeorGIA 30332-0160 USA

E-mail address: heil@math.gatech.edu

(K. Kornelson) Department of Mathematics, University of Oklahoma, Norman, OkLAHOMA 73019-0315 USA

E-mail address: kkornelson@math.ou.edu

(U. Molter) Departamento de Matemática, Facultad de Ciencias Exactas y Naturales, Universidad de Buenos Aires, Ciudad Universitaria, Pabellón I, 1428 Buenos Aires, Argentina and COniCEt, Consejo Nacional de Investigaciones Científicas y TÉCNicas, Argentina

E-mail address: umolter@dm.uba.ar 